\title{
IMPLICATIONS IN THE PHILOSOPHY OF SCIENCE FROM THE USE OF ARTIFICIAL NEURAL NETWORKS
}

\author{
Carlos Kazunari Takahashi ${ }^{1}$ and Marcos Amatucci ${ }^{2}$ \\ ESPM - Escola Superior de Propaganda e Marketing \\ ${ }^{1}$ Rua Dr. Álvaro Alvim, 123 - Vila Mariana, São Paulo - SP, 04018-010, Brazil \\ ${ }^{2}$ Rua Dr. Alvaro Alvim, no. 123 Sala C-404 - Vila Mariana, São Paulo - SP, 04018-010, Brazil
}

\begin{abstract}
The technological advance allowed new artificial intelligence algorithms to emerge. Among the new models, those of artificial neural networks are presented as one of the most disseminated, due to their accuracy in the prediction. However, this type of algorithm is commonly called the "black box", by using multiple obscure layers, rendering its logic-mathematical inaccessible and generating problems that affect the philosophy of science. The purpose of this paper is to present epistemological problems that emerge from the use of artificial intelligence and, more specifically, the artificial neural network (ANN) method.
\end{abstract}

\section{KEYWORDS}

Neural Networks, Artificial Intelligence, Philosophy of Science

\section{INTRODUCTION}

In March 2016, Alpha Go, artificial intelligence (AI) created by Google, was the first program to defeat the Go World Champion. From that date, a milestone was set in AI. Using artificial neural networks (ANN), Alpha Go achieved this achievement using only reinforcement learning, a dynamic ANN model that acts without the interference of human data (Hubert et al., 2017). The potential of using this technology as well as its epistemological and ethical implications is enormous (Bostrom, 2003; Bostrom e Yudkowsky, 2011). Human-insoluble problems can now be unraveled by this technology, which uses not only structured and supervised data to function, but also ANN (Russell et al., 2016).

Previous research (Horvitz, Breese e Henrion, 1988) already analyzed the implications of AI in social sciences, such as decision theory. However, the renewal of interest in this topic occurs when the evolution and dissemination of some algorithms, such as ANNs, change the way the postulates are understood. In addition, the present article also presents its relevance for seeking the integration between different areas of knowledge (Russell et al., 2016), aiming the advancement of the subject in academic scope. In this sense, the objective of this article is the presentation of epistemological problems emerged from the use of AI. Specifically, the implications of the artificial neural network (ANN) method on postulates.

Specifically, in the first part of the article, will be presented the advent of AI and its reflexes in studies of philosophy of science. Next, its implications will be presented in the philosophy of science. Finally, we will discuss some study alternatives that address the problems presented.

\section{BODY OF PAPER}

\subsection{Artificial Intelligence}

There are several definitions of AI in the literature (Faceli et al., 2011), from the most rational view, such "the study of the mental faculties through the use of computational models" (Charniak e McDermott, 1985) to the humanistic view, as "The art of creating machines that perform functions that require intelligence when 
performed by people" (Kurzweil et al., 1990). For this work, we will adopt the premise of action based on a rational agent that is one who acts to achieve the best result as possible (RUSSELL et al., 2016).

One of the techniques adopted in AI that use optimization is ANNs. By looking at the evolution of AI, the human brain is naturally based (Faceli et al., 2011; Haykin, 1994; Russell, Dewey e Tegmark, 2016). Although there has been some research since the 1940s (McCulloch e Pitts, 1943; Minsky e Papert, 1988), the ANN area began its full development only after the 1980, and only nowadays with the hardware and the big access to the datasets the field reborn (Faceli et al., 2011).

ANNs are distributed computing systems composed of densely interconnected processing units (Faceli et al., 2011). These units that compute mathematical functions can be called artificial neurons (Faceli et al., 2011), nodes or units (Russell et al., 2016). These are arranged in layers with multiple connections with associated weights, which ponder the importance of each artificial neuron in the network. These weights are adjusted as the learning process occurs, seeking optimization (Faceli et al., 2011).

The ANNs are presented as one of the most used methods currently for robotics and computer vision (Faceli et al., 2011). Its precision and tolerance for errors have made them widely used. On the other hand, one of his most constant criticisms is in understanding how ANN makes the decision. By using multiple hyperparameters, ANN develops very complex mathematical formulas for understanding and is commonly called "black box" (Faceli et al., 2011).

\subsection{Problems of the Advent of AI}

The growing development of AI research was not succeeded by equally important research in the Philosophy of Science on the subject (Bostrom e Yudkowsky, 2011). The creation of deep ANN algorithms tends to accelerate this process even more (Lecun, Bengio e Hinton, 2015) and, consequently, this disaggregation. Research on the Philosophy of Science in AI tends to be directed toward ethics as to the creation of a being who is aware of its existence (Allen, Wallach e Smit, 2006; Bostrom e Yudkowsky, 2011; Russell, Dewey e Tegmark, 2016) or to the capacity of the machine to act and interact as a human (McCarthy and Hayes, 1969).

One of the great works that pointed to AI problems was that of McCarthy and Hayes (1969), who, in analyzing the advance of AI up to that date, presented the frame problem. The problem arises when, in representing the dynamic world, in addition to explaining all the objects of study, one must also consider the rest as ceteris paribus, that is, other things being equal or assuming that nothing else interferes, but, in some way, was present at the event studied (Kincaid, 2008).

McCarthy and Hayes (1969) elaborated their work objectifying, in fact, the inflection point, where the machine could act and make decisions as humans. However, the frame problem also allows us to question current AI, both as regards ceteris paribus, and even the artifacts of causal action, that is, the agents themselves being analyzed.

Nor are Ceteris paribus undisputed in Philosophy. Kincaid (2008) argues that ceteris paribus assertions in the social sciences are a problem because they raise doubts about the claims they try to explain. The author claims that if we doubt the ceteris paribus mechanisms, even economic theories that use this premise may have their explanations put forward in contestation, and their predictions, just accidents of chance.

Causal explanations also touch on this problem. Among the alternative approaches that seek to explain "causation", those that use a counterfactual, considered by some researchers as the most promising (Woodward, 2008), have a great dependence on ceteris paribus.

Causal models are the basis of the logic behind ANN models, since they use a similar reasoning to generate predictions from the selected data (Haykin, 1994). Particularly in ANNs, the ceteris paribus contexts may be incomprehensible, since not necessarily all the parameters that must be kept constant for the realization of the forecast are known.

In this way, the first problem of AI, particularly of ANNs, is presented in the philosophy of science: (i) when assigning causal explanation and prediction in ANN models, how to understand and incorporate ceteris paribus contexts?

ANNs can also generate yet another critical problem in the philosophy of science. Until the technological advance of the 1990s, the use of regression and classification algorithms worked with a logical-mathematical structure accessible to researchers, where it was possible, in a trivial way, to list the important variables for the model, as well as their respective weighting. 
With the return of ANNs, together with the increase in computational power, new algorithms with increasing numbers of variables and even deeper layers were introduced (Lecun, Bengio and Hinton, 2015), generating very complex and incomprehensible models. According to Faceli et al. (2011), the knowledge generated for the explanation and prediction of ANNs is stored in many parameters arranged in inaccessible mathematical equations. Therefore, they are commonly referred to as black boxes.

This event has repercussions on the philosophy of science, since, in addition to the ceteris paribus contexts, not even the subject variables of action are well known nor their individual contribution to the phenomenon. As a result, we can identify the second problem: (ii) when using ANN models, how to understand the intrinsic parameters of these highly complex contexts in order to be able to unequivocally attribute causal explanation and prediction?

In May 2018, the European Parliament instituted the General Data Protection Regulation (GDPR) and, among the various aspects of this regulation, clauses on automated decision-making are included, which focuses on ANNs, where there is an individual right to an explanation on the logic involved in decision making (Guidotti et al., 2018). Thus, the search for correspondence of these questions is emerging, not only in an academic context, but also for practical purposes.

\subsection{Alternatives for the Elucidation of AI Problems}

A more adequate form of causation analysis is one of the most important items on the agenda for future work on causal explanation (Woodward, 2008). After presenting the problems of AI that focus on the philosophy of science and that reside in the complexity of interpretation, often in real time, of the algorithms of ANN, will be presented some alternative lines of searches for solving the problems.

A common goal for both questions is the search for greater transparency in their predictions. Both the understanding of the ceteris paribus of a given analysis and the weights and amounts of variables in others are determinant for the validity of the studies.

For the first problem, in the ANN models, the ceteris paribus instances are treated as model errors, that is, other facts that influence the model, but that the algorithm cannot interpret with the data that it possesses. In this way, it is possible to know that there are other things influencing the phenomenon. We know how much the sums of these instances are influencing the model, but we do not know what these variables are, nor their individual weighting. About this problem, little study has been done, and no alternative was found. One way to improve this approach comes with the introduction of methods that interpret unstructured data captured by web crawling, or devices that "sweep" the internet for data, but this approach is still in its infancy.

For the second problem, some alternatives are presented seeking the interpretation of the data. The study presented by Guidotti et al. (2018) carried out an extensive research on methods of analysis of "black-box" models. The authors found three methods of interpretation: the extraction of the parameters by a set of rules, by a decision tree or by the creation of a prototype. However, despite the extensive analysis, research on the interpretation of ANN data is still presented as open-ended problems (Guidotti et al., 2018; Mahendran e Vedaldi, 2015) and there is no agreement on what an explanation is GUIDOTTI et al., 2018.

It is evident that the research activity in this field is not providing yet a sufficient level of importance in the study of general and common formalism for defining an explanation, identifying which are the properties that an explanation should guarantee, soundness, completeness, compactness and comprehensibility. Concerning this last property, there is no work that addresses the problem of quantifying the grade of explanation for humans, although it is of fundamental importance (GUIDOTTI et al. 2018).

Therefore, the need for more complex analyzes that address these issues is evident, and the philosophy of science, in a prominent way, can help to build the bases of understanding of the problems of ANNs.

\section{CONCLUSION}

In the present article, the problems that the advent of ANNs have repercussions in the philosophy of science were presented. Initially, we exposed the context of the emergence of AI and its evolution over the decades. Next, we focus on one of the types of AI, the ANN, and the consequence of their development, generating new impacts on the philosophy of science. Finally, some alternative solutions to the problems denoted were offered, despite their incipient development. 
As presented in the article, no solution was developed for the first problem: "(i) when assigning causal explanation and prediction in ANN models, how to understand and incorporate ceteris paribus contexts?" As for the second, "(ii) when using ANN models, how to understand the intrinsic parameters of these highly complex contexts to be able to attribute, undeniably, causal explanation and prediction?", Some decoding alternatives were presented, but no representation solution.

However, developing AI algorithms, both ANN and others, requires compromises on what knowledge is and how it is obtained (McCarthy and Hayes, 1969). This need for transparency lies not only in the academic sphere, but changes in data protection regulations (Guidotti et al., 2018) also reinforce the need for further study and development in this field.

Therefore, the AI area presents itself as a fruitful field for the development of epistemological research, either because of its disruptive development, which creates the need for new perspectives on previously well-defined themes, or the need for a human approach, which must define guidelines for the development of AI.

\section{ACKNOWLEDGEMENT}

This study was financed in part by the Coordenação de Aperfeiçoamento de Pessoal de Nível Superior - Brasil (CAPES) - Finance Code 001

\section{REFERENCES}

Allen, C.; Wallach, W.; Smit, I. IEEE Intelligent Systems Why Machine Ethics? IEEE Intelligent Systems, n. July/August, p. $12-17,2006$.

Bostrom, N. Ethical Issues in Advanced AI. Cognitive, emotive and ethical aspects of decision making in humans and in artificial intelligence, v. 2, p. 12-17, 2003.

Bostrom, N.; Yudkowsky, E. The ethics of artificial intelligence. Cambridge University Press, 2011.

Charniak, E.; Mcdermott, D. Introduction to Artificial Intelligence Programming. [s.1: s.n.].

Faceli, K. et al. Inteligência Artificial: Uma abordagem de aprendizado de máquina. 2011.

Guidotti, R. et al. A Survey Of Methods For Explaining Black Box Models. v. 51, n. 5, 2018.

Haykin, S. Neural networks: a comprehensive foundation. [s.l.] Prentice Hall PTR, 1994.

Horvitz, E. J.; Breese, J. S.; Henrion, M. Decision theory in expert systems and $\{\mathrm{AI}\}$. Intl. J. Approximate Reasoning, v. 2, n. 3, p. 247-302, 1988.

HuberT, T. et al. Mastering the game of Go without human knowledge. Nature, v. 550, n. 7676, p. 354-359, 2017.

Kinkaid; H. Social Sciences. In: Silberstein, M. The Blackwell Guide to the Philosophy of Science, 2008

Kurzweil, R. et al. The age of intelligent machines. [s.l.] MIT press Cambridge, MA, 1990. v. 579

Lecun, Y.; Bengio, Y.; Hinton, G. Deep learning. Nature, v. 521, n. 7553, p. 436-444, 2015.

Mahendran, A.; Vedaldi, A. Understanding deep image representations by inverting them. Proceedings of the IEEE conference on computer vision and pattern recognition. 2015

Mccarthy, J.; Hayes, P. J. Some Philosophical Problems from the Standpoint of Artificial Intelligence. In: Readings in Artificial Intelligence. [s.1.] Elsevier, 1969. p. 431-450.

Mcculloch, W. S.; Pitts, W. A logical calculus of the ideas immanent in nervous activity: The bulletin of mathematical biophysics. Bulletin of Mathematical Biophysics, v. 5, n. 4, p. 115-133, 1943.

Minsky, M. L.; Papert, S. A. Perceptrons. 1988.

Russell, S. J. et al. Artificial Intelligence a Modern Approach Third Edition, 2016.

Woodward; J. Explanation. In: Silberstein, M. The Blackwell Guide to the Philosophy of Science, 2008. 